\title{
Conversion of multi-layered Mg-based carrier to hydrogen storage performance
}

\author{
Ningning Zhou ${ }^{a \cdot b}$ and Dongying Jub* \\ aPetrochemical Research Institute, Liaoning Petrocchemical Vocational and Technology, No. 4, District 2 Beijing Road, Jinzhou, Liaoning, \\ 121001, China \\ ${ }^{b}$ Department of Electronic Engineering, Saitama Institute of Technology, Fukaya, Saitama 369-0293, Japan
}

\begin{abstract}
The mixed powder method and covering method were adopted to prepare a composite that has hydrogen storage properties. In the experimental process, the alloy phases $\mathrm{Mg}_{2} \mathrm{Ni}$ and NiTi generated regularly; the penetration of the hydrogen atoms to the material was considered as the adsorption of the material to the hydrogen atoms; the original thermodynamic equation was edited; the crystal phases were optimized by adjusting the parameters about the material composition and gas concentration, so that providing advantageous channels of the hydrogen atoms in and out to improve the hydrogen storage properties. The results showed that integer multiples adjustment to trace element Ti could make hydrogen storage properties maximizing optimize and have controllability for the capacity and rate of hydrogen storage according to the newly editing equation. The innovation was to edit out equation that can improve the hydrogen storage properties through adjusting three parameters of trace element $\mathrm{Ti}$, adsorption and penetration to find the most optimal condition improving the hydrogen storage properties.
\end{abstract}

\section{Introduction}

In recent years, the fossil fuel reserves are rapidly reduced lead to the existing reserves cant not meet the growing demand. The majority of the world energy currently comes from the fossil fuels, but the use of the fossil fuels can produce large amounts of the harmful substances, which causes a great threat to the environment. Therefore, accelerating the conversion of the energy system to renewable energy meets the energy needs of the current and future world has become urgent problem to be solved. ${ }^{[1-4]}$ In particular, how to adopt more efficient functional materials and to effectively control hydrogen absorption and desorption under a certain condition have been main research directions. Expanding applied range of hydrogen storage material, developing various potential functional, particularly, the using of the hydrogen storage media have became concern issues. In the present work, low price, abundant resources and light quality $\mathrm{Mg}$ based material was adopted for two-way development of hydrogen storage material. A new type prepared techniques included that synthesis-formingsintering-alloying achieved crystalline phase restructuring to generate the composite of clad $\mathrm{Mg}_{2} \mathrm{NiX}$-NiTiX (watchcase)- pure $\mathrm{Mg}$ (inner casing). To distinguish conventional methods, the advantages from the powder and alloy were synthesized by covered method to achieve the adding of the powder to the metal and the alloy phases $\mathrm{Mg}_{2} \mathrm{Ni}$ and NiTi generated continuously from the surface to the inner. Through activated treatment on the surface, crystalline phase characteristic and organizational structure were improved and optimized.

\section{Experimental results and discussion}

\subsection{Actual results before adjusting Ti content}

As shown in Figure 1a, the composite at the molar ratios of $\mathrm{Ni}: \mathrm{Ti}: \mathrm{Mg}=\beta: \gamma: \eta$ absorbed hydrogen relatively slowly under the constant pressure and constant temperature of $340^{\circ} \mathrm{C}$, with the increasing of hydrogen pressure, hydrogen absorption tended to increase slowly. The saturation produced when the hydrogen absorption reached $2.6 \%$. Hydrogen desorption started with the changes of hydrogen pressure for 6 hours after reaching saturation, the actual capacity of hydrogen desorption was $2 \%$. As shown Figure 1b, the proportion of the materials had greater effect on the hydrogen storage performance based on the directions of present experiment. There were no obvious changes about the capacity of hydrogen absorption (in unit of $/ \mathrm{mg}$ ) within the 35 hours area, the initial activation and the concentration of the alloy was relatively lower. It indicated that the effective area was relatively small due to the insufficient reaction of the powder with pure $\mathrm{Mg}$ ingot or the insufficient unit content of a certain element. It is considered that the addition of powder can effectively control the surface area. The addition of powder Ni was definite purpose in this experiment. The

\footnotetext{
${ }^{a}$ Corresponding author: zhouningning_yes@163.com
} 
addition of $\mathrm{Ni}$ powder at a certain ratio enable the reaction with pure $\mathrm{Mg}$ enhanced to generate $\mathrm{Mg}_{2} \mathrm{Ni}$ alloy phase. If any deviation produced, the $\mathrm{MgNi}_{2}$ alloy phase will be generated and it will prevent hydrogen absorption and adsorption. Therefore, the added amount of powder $\mathrm{Ti}$ is controllable. Figure 1c showed that the penetrated capacity of the hydrogen to the material is the key factor in the process of the hydrogen permeated to the composite under the temperature of $340^{\circ} \mathrm{C}$ (the adsorption of the material to $\mathrm{H}_{2}$ was considered as the penetration of $\mathrm{H}_{2}$ to the material). If the activation time did not considered, the formula as shown in

$$
\Pi=C \times R \times T
$$

Mis the permeability of hydrogen; $\mathrm{C}$ is a penetrated concentration; $\mathrm{R}$ is a gas constant; $\mathrm{T}$ is an absolute temperature. According to a amount of $\mathrm{H}$ atoms, it was considered as the absorbed capacity of the material after penetrating to the material, the maximum permeate pressure reached $6500 \mathrm{mOsm} / \mathrm{Kg}$.

The rate of hydrogen absorption and desorption showed the materials used in this experiment was hard to activate. In terms of materials, $\mathrm{Mg}_{2} \mathrm{Ni}$ alloy phase can absorb sufficiently hydrogen atoms but its hydrogen absorption rate is relatively slow, NiTi alloy phase can effectively provide $\mathrm{H}$ atoms to enter channel. The above data suggested that the generation of NiTi was insufficiently. Therefore, the adjustment of $\mathrm{Ti}$ as a key can improve the hydrogen absorption and adsorption properties.

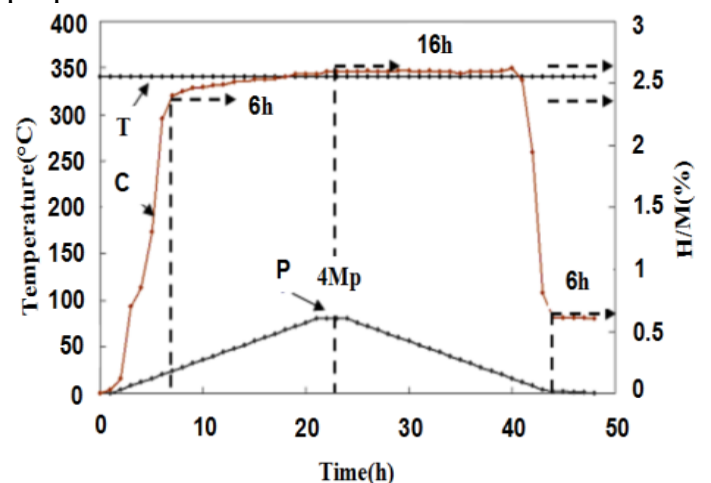

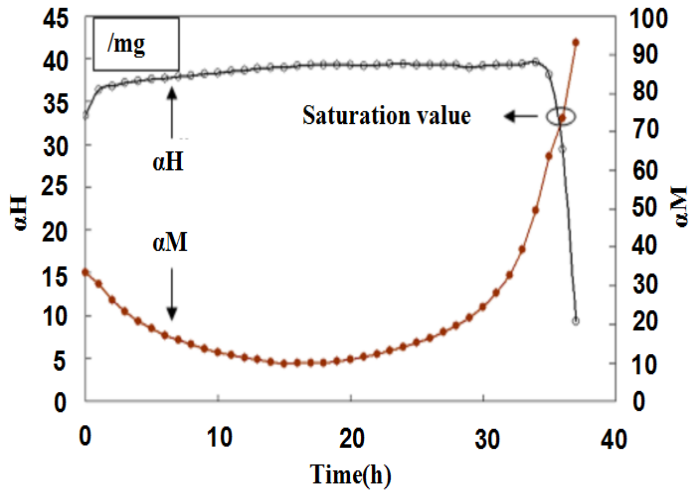

$\mathrm{b}$

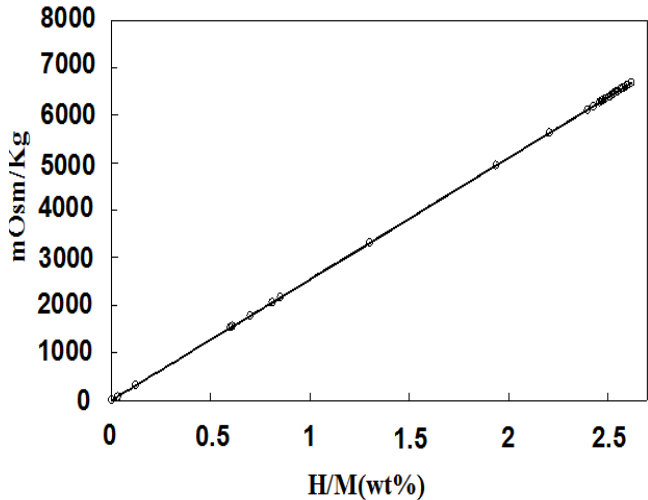

c

Figure 1. Analysis and calculation of P-C-T curve before adjusting Ti. 1. P-C-T curve under constant temperature $340^{\circ} \mathrm{C}$. 2. Relative concentrations between $\mathrm{H}$ atoms and alloy. 3. Actual relation between the permeate pressure of $\mathrm{H}_{2}$ to the material and the concentration of the material absorbed $\mathrm{H}_{2}$ before adjusting Ti content.

\subsection{Simulated result after adjusting Ti content}

The research purpose was to improved the hydrogen absorption and desorption ability under a lower temperature $220^{\circ} \mathrm{C}$ by adjusting the concentration of the hydrogen. In order to confirm the consistency of the simulated value and actual value after the absorbed capacity, the hydrogen absorption ability of the material to the hydrogen was considered as the penetration of hydrogen to the material. According to Ti atom could enter into face centered cubic position, two Ni-Ti compound bonds might be form in an aeromonas with the addition of $\mathrm{Ti}$ at molar amount. NiTi alloy phase provided the channel of $\mathrm{H}$ atoms entered into the material. Therefore, the concentration of $\mathrm{H}$ would be expand 2 times (analog value) $\left[\mathrm{C}_{\mathrm{H}}\right.$ (Fig. 1a) $\left.\times 2\right]$ according to $\Pi=C \times R \times T$, the simulated result showed that the permeate ability of hydrogen to the material could be improved obviously with the increasing of the hydrogen concentration, so that the permeate pressure reached $10656.89 \mathrm{mOsm} / \mathrm{Kg}$ in Figure 2. 


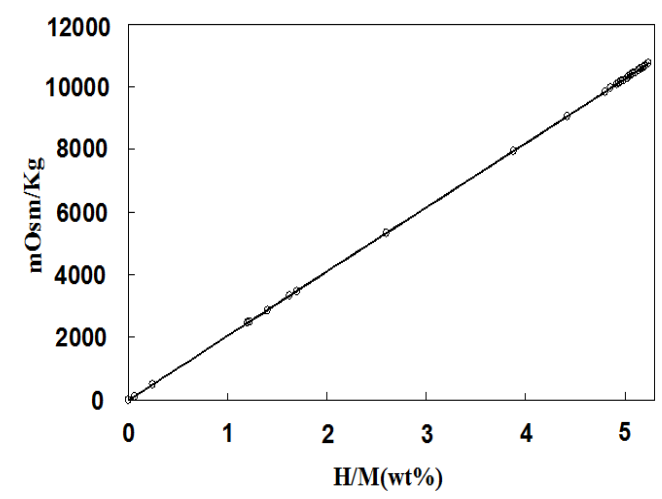

Figure 2. Simulated relation between the permeation of $\mathrm{H}_{2}$ to the material and the concentration of the material absorbed $\mathrm{H}_{2}$ after adjusting Ti content.

\subsection{Actual results on substituted into revised formulas after adjusting Ti content}

The study conducted structure-recombination in sintered process, the materials were constituted at the molar ratio of $\mathrm{Ni}: \mathrm{Ti}: \mathrm{Mg}=\beta:(\gamma+1): \eta$ under the constant temperature of $220^{\circ} \mathrm{C}$, which is far below $300^{\circ} \mathrm{C}$. The actual verified result was confirmed by adjusting the component and revising the formulas. The original formula and the specific revised process as shown in follow

$$
\ln p=\frac{\Delta H}{R T}-\frac{\Delta S}{R}
$$

Where $\mathrm{P}$ is equilibrium pressure; $\triangle \mathrm{H}$ is the endothermic enthalpy; $\triangle S$ is the endothermic entropy; $T$ is the absolute temperature; $\mathrm{R}$ is the gas constant, through extending the original formula, the relationship formula between formed the metal hydrides and the metal is discussed

$$
K_{p}=\frac{\alpha_{M H_{X}}}{\alpha_{M}\left(f_{H_{2}}\right)^{\frac{X}{2}}}
$$

Where $\mathrm{K}$ is the equilibrium constant; $\alpha$ is substance activity; $\mathrm{M}, \mathrm{MH}$ is the standard state; $\alpha \mathrm{MH}$ and $\alpha \mathrm{M}$ are activity; $\mathrm{f}$ is gas fugacity. In which the equilibrium constant Kp equal to

$$
K_{p}=P_{H_{2}}
$$

The formulas could be changed to

$$
P_{H_{2}}=\frac{\alpha_{M H}}{\alpha_{M}\left(f_{H_{2}}\right)^{\frac{X}{2}}}\left(f_{H_{2}} \approx 1\right)
$$

$\mathrm{P}_{\mathrm{H} 2}$ is equilibrium hydrogen pressure. In the formulas, $\mathrm{f}_{\mathrm{H} 2}$ is considered as 1 , therefore, the equilibrium $\mathrm{H}_{2}$ pressure is related to the concentration of metal hydride and the pure metal, as shown in below

$$
P_{H_{2}}=\frac{\alpha_{M H}}{\alpha_{M}}
$$

In order to macroscopic analysis the effect of introducing components on the hydrogen storage, the capacity of the metal hydride after the cycle of hydrogen absorption and desorption became seldom and uncertain. $\alpha \mathrm{M}$ was approximately equal to the concentration of the pure metals, the metal can be divided to three kinds of pure metal $\alpha \mathrm{Mg}, \alpha \mathrm{Ni}$ and $\alpha \mathrm{Ti}$, respectively.

$$
\alpha_{M} \approx \alpha M g_{\frac{\eta}{24}}+\alpha N i_{\frac{\beta}{59}}+\alpha T i_{\frac{r}{48}}
$$

Substituting into formula (2.4)

$$
P_{H_{2}}=\frac{\alpha_{H}}{\alpha M g_{\frac{\eta}{24}}+\alpha N i_{\frac{\beta}{59}}+\alpha T i_{\frac{r}{48}}}
$$

$\eta$ is the quality of $\mathrm{Mg}$; $\beta$ is the quality of $\mathrm{Ni} ; \gamma$ is the quality of Ti. Therefore, the adding of trace element $\mathrm{Ti}$ can be adjusted according to the formulas

$$
\alpha_{M} \approx \alpha M g_{\frac{\eta}{24}}+\alpha N i_{\frac{\beta}{59}}+\left(\alpha T i_{\frac{r}{48}}+\alpha T i_{\frac{\varepsilon}{48}}\right)
$$

So the below formula is obtained

$$
P_{H_{2}}=\frac{\alpha_{M H}}{n \alpha_{M}}
$$

$\mathrm{n}$ is a alloy phase concentration factor. It is considered that the hydrogen pressure had relationship with the component of adding the element, the performance of hydrogen absorption and desorption can be improved by adjusting the component of the raw material. Accordance to above export formula, the absorption capacity of hydrogen atoms was viewed as the gas permeation. $\alpha_{\mathrm{MH}}$ is deformed into $\alpha_{H}$ as shown in follow

$$
P_{H_{2}}=\frac{\alpha_{H}}{n \alpha_{M}}
$$

In order to avoid the deviation from traditional calculated method, the absorbed hydrogen concentration is regarded as the permeability ability of gas $\mathrm{H}_{2}$. The below formulas can be obtained

$$
\alpha_{H}=C_{H}=\frac{\Pi}{R T} \approx m \frac{\Pi}{R T}
$$

$\Pi$

$\overline{R T}$ is the gas permeability. Through adjusting the adding of trace element $\mathrm{Ti}, \mathrm{H}_{2}$ permeability had been validated according to the below formulas

$$
P_{H_{2}}=\frac{m \frac{\Pi}{R T}}{n \alpha_{M}}(n \approx m)
$$

$\mathrm{n}$ and $\mathrm{m}$ are concentration factors and the differ are small, the value of $\mathrm{n}$ and $\mathrm{m}$ approximately equal 2 . It is indicated that effectively control the content of $\mathrm{Ti}$ can provides a powerful channel for hydrogen absorption and desorption. The formula (3.1) is substituted into (2.1) and (2.2)

$$
P_{H_{2}}=\frac{m \frac{\Pi}{R T}}{n \alpha_{M}\left(f_{H_{2}}\right)^{\frac{X}{2}}}(m \approx n)
$$




$$
\Pi=\frac{P_{H_{2}} \times n \alpha_{M}\left(f_{H_{2}}\right)^{\frac{X}{2}} \times R T}{m}(m \approx n)
$$

As shown Figure 3a, the hydrogen absorption speed was improved; hydrogen absorption amounted to $3 \%$ within 3 minutes and 5\% within 16 minutes. Not only the temperatures for hydrogen absorption had been reduced significantly, but also the amount and the speed of hydrogen absorption had been also improved significantly. As shown Fig. 3b, the hydrogen absorption reached quickly the saturation value and the concentration of the alloy (in unit of /g) was relatively lower, it indicated the concentration of hydrogen had enhanced within the relatively short time. As shown in Fig. 3c, the concentration of Ti was adjusted to verify and simulate the permeation of the hydrogen to the material due to the concentration of hydrogen could not be controlled through the simulation. According to the hydrogen atom enter into the composite in form of tetrahedral or octahedral gap, the molar ratio was adjusted to $\mathrm{Ni}: \mathrm{Ti}: \mathrm{Mg}=\beta:(\gamma+1): \eta$, it is considered that the effective increasing of $\mathrm{Ti}$ content could form fully NiTi alloy phase in the sintered process and reduce the loss of the reaction from $\mathrm{Ni}$ and $\mathrm{Mg}$, which provided more channels for hydrogen absorption/desorption. To increase the molar amount of $\mathrm{Ti}$, the ideal state was each $\mathrm{Ti}$ atom and $\mathrm{Ni}$ atom reacted to generate $\mathrm{NiTi}$, so that effectively increasing the surface of the composite material and improving the performance of hydrogen absorption/desorption. According to this theory, the formulas would be revised and obtained verification, the maximum permeated pressure reached to $11005.28 \mathrm{mOsm} / \mathrm{Kg}$ by the revised formula (3.3). This result was consistent with above simulated result of $10656.89 \mathrm{mOsm} / \mathrm{Kg}$; it was showed that adjusting $\mathrm{Ti}$ content could achieve controllability of the absorbed capacity of the material considered as the penetration of the hydrogen according to revise formulas.

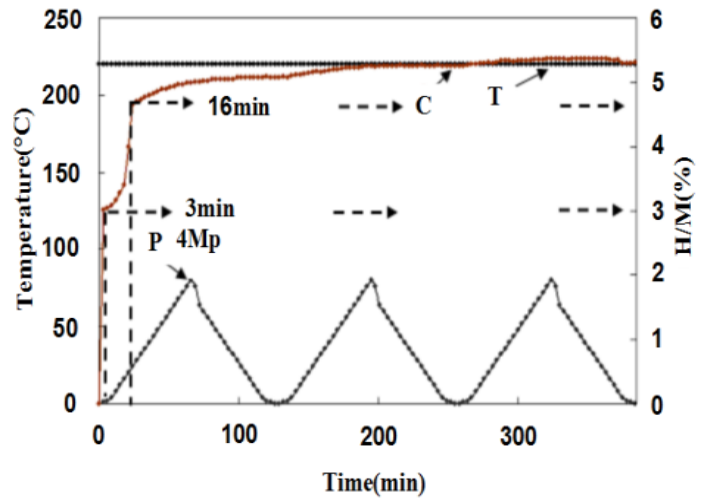

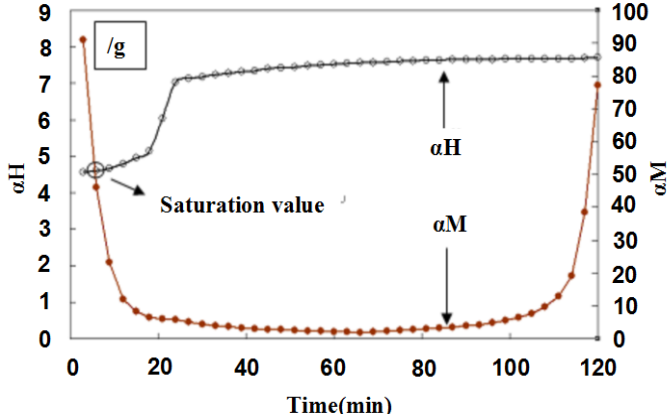

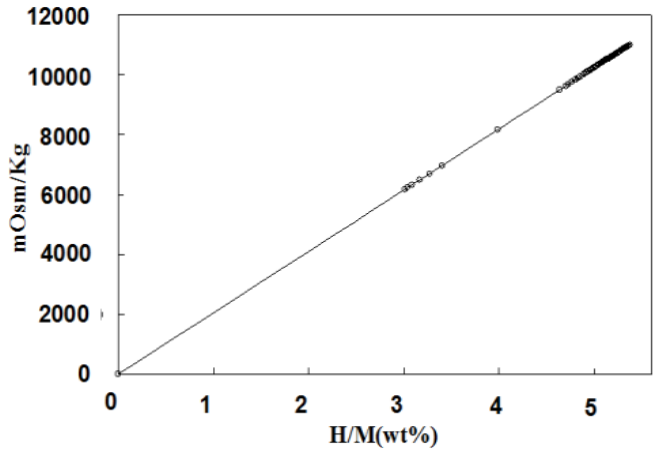

Figure 3. Analysis and calculation of P-C-T curve after adjusting Ti. 1. P-C-T curve under constant temperature $220^{\circ} \mathrm{C}$. 2. Relative concentrations between $\mathrm{H}$ atoms and alloy. 3. Actual relation between the permeate pressure of $\mathrm{H}_{2}$ for the material and the concentration of the material absorbed $\mathrm{H}_{2}$ after adjusting Ti content.

The results from the actual operation and simulation were consistence,the absorbed channel of the hydrogen atom could be increased effectively through adjusting the concentration of $\mathrm{Ti}$, and original formula would be revised by the new method that adjusting the concentration of the original material. This actual result was closed to that after simulating. Adjusting the concentration of $\mathrm{Ti}$ and simulating concentration of hydrogen was basically consistent.

\section{Conclusion}

In this work, the composite effectively overcame inconvenient from the powder on storage aspect and avoided negative factors of small surface area due to an increasing of the grain boundary between the crystal grains and the channels of $\mathrm{H}$ entered into the material increased. For the composite, hydrogen absorption and desorption rate was obviously improved and even superior to the effects from a powder by traditional method. The composite had improved greatly the capacity and rate of hydrogen absorption/desorption. The capacity of hydrogen absorption reached 3\% within 3 min and the highest amount reached $5.3 \%$ within $16 \mathrm{~min}$. In this study, the adjusting of Ti had a certain range, what effects will produce if the proportion will become excessive need to be further researched.

\section{References}

[1] D. Kyoi, T. Sakai, N. Kitamura, A. Ueda and S. Tanase, Synthesis of FCC Mg-Ta Hydrides Using GPa Hydro-gen Pressure Method and Their Hydrogen- 
Desorption Properties. ALLOYS COMPOUNDS. J. 463, 306-310 (2008).

[2] A. Singh, B.K. Singh, D.J. Davidson and O.N. Srivastava, Studies on improvement of hydrogenstorage capacity of AB5 type: $=\mathrm{MmNi4} .6 \mathrm{Fe} 0.4$ alloy. HYDOGEN ENERG. J. 29, 1151-1156 ( 2004).

[3] L. Schlapbach, A. Züttel, Hydogen-Storage Matcrials for Mobile Application. Journal of Nature. J. 414,353358 (2001)

[4] M. V. Simičić, M. Zdujić, R. Dimitrijević, L. Nikolić-Bujanović, N. H. Popović, Hydrogen Absorption and Electrochemical Properties of $\mathrm{Mg}_{2} \mathrm{Ni}$-Type Alloys Synthesized by Mechanical Alloying. J POWER SOURCES. J. 158, 730-734 (2006). 